\title{
LmxMPK4, a mitogen-activated protein (MAP) kinase homologue essential for promastigotes and amastigotes of Leishmania mexicana Qiong Wang ${ }^{\dagger}$, Inga M Melzer ${ }^{\dagger}$, Martin Kruse, Claudia Sander-Juelch and Martin Wiese*
}

Address: Bernhard Nocht Institute for Tropical Medicine, Parasitology Section, Bernhard-Nocht-Strasse 74, D-20359 Hamburg, Germany Email: Qiong Wang - qiongkunle@yahoo.com; Inga M Melzer - inga.melzer@bni-hamburg.de; Martin Kruse - martin.kruse@zmnh.unihamburg.de; Claudia Sander-Juelch - juelch@bni.uni-hamburg.de; Martin Wiese* - martin.wiese@bni-hamburg.de

* Corresponding author †Equal contributors

Published: 29 December 2005

Kinetoplastid Biology and Disease 2005, 4:6 doi:10.1 186/1475-9292-4-6

This article is available from: http://www.kinetoplastids.com/content/4/I/6

(C) 2005 Wang et al; licensee BioMed Central Ltd.

This is an Open Access article distributed under the terms of the Creative Commons Attribution License (http://creativecommons.org/licenses/by/2.0), which permits unrestricted use, distribution, and reproduction in any medium, provided the original work is properly cited.
Received: 21 October 2005

Accepted: 29 December 2005

\begin{abstract}
Background: Leishmania parasites undergo profound morphological and biochemical changes while passing through their life cycle. Protein kinases have been shown to be involved in the differentiation from the extracellular flagellated promastigotes to the intracellular "non-flagellated" amastigotes and vice versa. Moreover, these enzymes are likely involved in the regulation of the proliferation of the different life stages.
\end{abstract}

Results: Here, we characterize LmxMPK4, a mitogen-activated protein (MAP) kinase homologue from Leishmania mexicana. The kinase reveals all sequence motifs for classification as a MAP kinase. LmxMPK4 proved to be active as a recombinant protein. The kinase is expressed in promastigotes and amastigotes. It was impossible to generate homozygous gene deletion mutants for LmxMPK4 in promastigotes. Moreover, amastigotes bearing only an episomal copy of the gene stably retained LmxMPK4 over a prolonged period without antibiotic pressure in infected mice.

Conclusion: LmxMPK4 is essential for promastigotes and amastigotes of Leishmania. It shows significant amino acid sequence divergence to mammalian MAP kinases. Thus, LmxMPK4 is a promising new drug target.

\section{Background}

Protein kinases are key regulatory molecules in all eukaryotic cells. Together with their antagonists the protein phosphatases they form complex networks of mutually activating and silencing molecules. Their activity affects vital processes like differentiation, proliferation, motility, stress response, and apoptosis [1]. Mitogen-activated protein (MAP) kinases are often the final kinases in signal transduction cascades relaying signals augmented by environmental stimuli via multiple steps of reversible phosphorylation from different receptors to effector proteins which can ultimately lead to changes in protein expression profiles. In higher eukaryotes the core of the MAP kinase signal transduction cascade is comprised of a MAP kinase kinase kinase (MKKK), a MAP kinase kinase (MKK) and a MAP kinase. Upon a stimulus the MKKK is activated by phosphorylation. The active MKKK phosphorylates a MAP kinase kinase (MKK) on serine and/or threonine residues in its phosphorylation loop. MKKs are dual-specificity kinases which are able to phosphorylate their substrates, the MAP kinases, on a threonine and a tyrosine residue of the conserved TXY motif of the phosphoryla- 

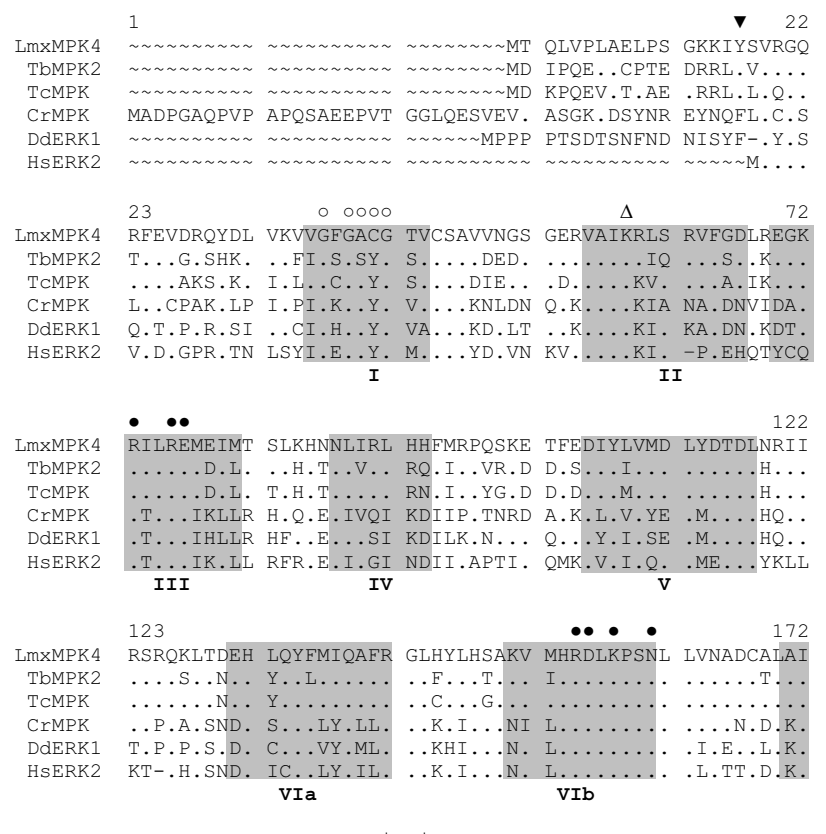

172

LmXMPK 4

TbMPK2

TCMPK

CrMPK

DdERK1

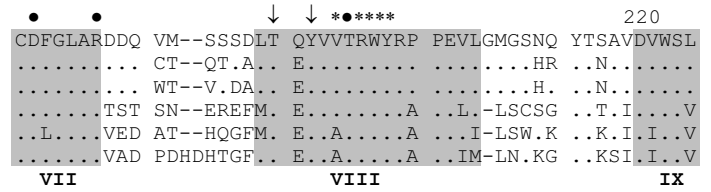

VII

VIII

267

LmXMPK4

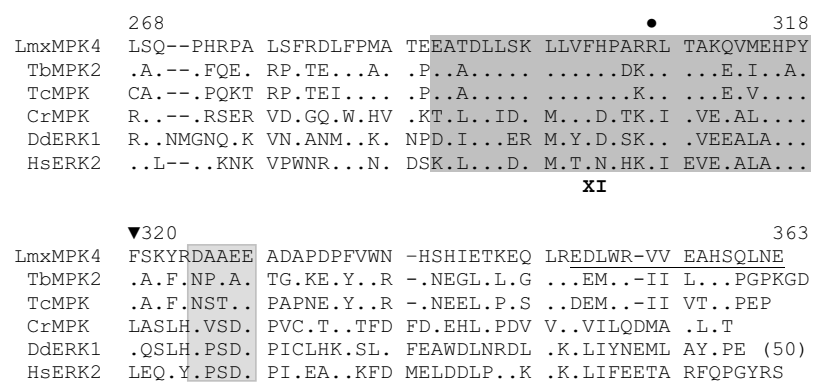

Figure I

Alignment of LmxMPK4 from $L$. mexicana with various MAP kinase amino acid sequences. LmxMPK4, Leishmania mexicana MAP kinase 4 (GenBank accession number AJ293282; [6]); Trypanosoma brucei MAP kinase, TbMAPK2 (GeneDB accession number Tb 10.70.2070; [ I0]); Trypanosoma cruzi MAP kinase (GeneDB accession number Tc00.10470535I I 299.70); Chlamydomonas reinhardtii MAP kinase (GenBank accession number BABI827I); Dictyostelium discoideum ERKI (GenBank accession number UI 1077; [ 13]); Homo sapiens ERK2 (GenBank accession number CAA77753; [I4]). Roman numerals I to XI indicate kinase subdomains. The kinase domain is located between the two inverted triangles ( $\nabla)$. Arrows $(\downarrow)$ mark the potential regulatory phosphorylation sites in the TXY motif at Thr 190 and Tyr 192, filled circles (O) indicate conserved amino acid residues it is not clear what you mean by conserved aa residues, and open circles $(\bigcirc)$ depict the residues of the phosphate anchor ribbon for ATP binding. The asterisks (*) show the residues of the P+I specificity pocket. The open triangle $(\Delta)$ marks the invariant lysine residue (K59), which is essential for the phosphate transfer reaction. The CD-domain is shaded in light grey. Dashes indicate gaps introduced to optimize the alignment; dots represent identical residues. The carboxy-terminal peptide used to generate antibodies is underlined. (50), the terminal 50 amino acid residues of the Dictyostelium kinase have been omitted. Numbering corresponds to LmxMPK4. 


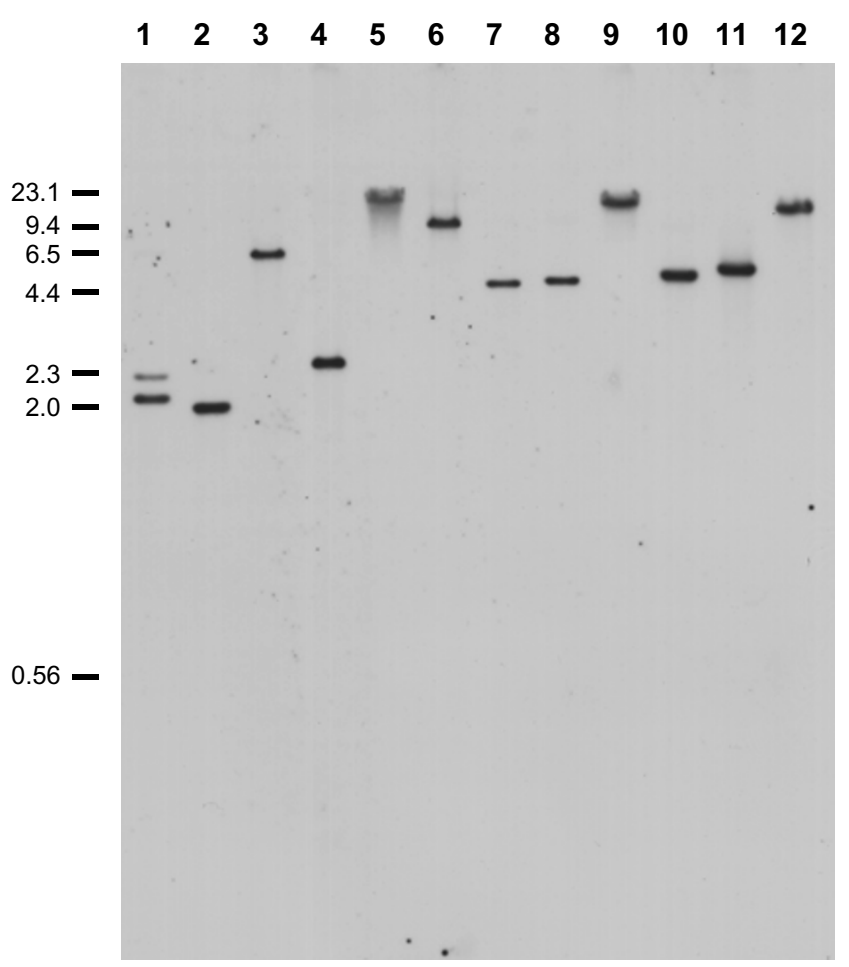

Figure 2

Southern analysis of genomic DNA. I, Pstl; 2, Hincll; 3, HindIII; 4, Acc65I; 5, Xbal; 6, Spel; 7, EcoRV; 8, Clal; 9, Ndel; I0, Ncol; II, Mlul; I 2, Munl. Digested DNA (5 $\mu \mathrm{g}$ ) was electrophoresed on a $0.7 \%$ agarose gel, blotted onto nylon membranes and probed with a DIG-labelled DNA probe corresponding to a fragment of the open reading frame ( $\mathrm{nt}$ 240 - nt 930). Numbers indicate the approximate size of DNA markers in kb.

tion loop in order to cause maximal enzymatic activity. Finally, activated MAP kinases can translocate into the nucleus and phosphorylate transcription factors or phosphorylate cytosolic kinases or structural proteins. Genome sequencing and analysis of Leishmania major and L. infantum revealed the presence of all members of the MAP kinase signal transduction core module [2]. However, neither typical receptors that lead to the activation of MAP kinase signal transduction pathways like receptor tyrosine kinases and G-protein coupled receptors nor typical MAP kinase substrates like transcription factors for RNA polymerase II promoters have been found in the parasites. It is not known yet how regulation of gene expression is achieved in the parasites, but it is likely that the substrates of MAP kinases are gene regulatory molecules, albeit not transcription factors. It is generally assumed that regulation occurs on post-transcriptional levels like the maturation of the mRNA from a polycistronic precursor to a capped and poly-adenylated mRNA in trans splicing, the mRNA stability, the efficiency of translation or even the protein stability.
Leishmania parasites have a digenetic life cycle with mammals and sand flies as their hosts. While passing through their life cycle the parasites alternate between proliferative forms, the procyclic promastigotes and the amastigotes, and forms arrested in the cell cycle, the highly infectious metacyclic promastigotes. Protein kinases are likely involved in the regulation of the proliferation of the different life stages and in the differentiation processes from procyclic promastigotes to metacyclic forms, to amastigotes after transmission to a mammal and uptake by host macrophages, and back to promastigotes once the amastigotes reach the gut of a sand fly. Members of the MAP kinase signalling pathways have been found to play important roles in differentiation and proliferation of Leishmania. LmxMKK a MAP kinase kinase homologue is involved in the formation and maintenance of the flagellum in the promastigote. A null mutant displayed flagella reduced to maximally $1 / 5$ of the length of the wild type flagellum [3]. It is likely that this kinase is involved in the outgrowth of a new flagellum prior to cell division in the promastigotes and in the differentiation from the amastigote displaying a short flagellum not protruding from the flagellar pocket, an invagination of the plasma membrane at the base of the flagellum, to the promastigote with a flagellum which could even be longer than the body of the cell. With LmxMPK9 a second kinase belonging to a MAP kinase signalling pathway has been found which is involved in the regulation of flagellar length. Here, null mutant promastigotes showed elongated flagella as compared to wild type cells, indicating that this kinase affects the shortening of the flagellum [4]. LmxMPK1, the first MAP kinase homologue described in L. mexicana, has been found to be essential for the proliferation of the amastigotes [5]. A deletion mutant was still able to infect host cells, transform to the amastigote morphology, but was unable to proliferate. As such, this kinase is an ideal drug target and a specific inhibitor would be promising to treat the disease.

Here, we characterize LmxMPK4, a MAP kinase homologue from L. mexicana, which is essential for promastigotes and amastigotes, and consequently also has the potential to be used as a drug target to treat Leishmaniasis.

\section{Results}

\section{Molecular characterisation of LmxMPK4}

We cloned and sequenced the gene for LmxMPK4, which is a MAP kinase homologue of L. mexicana. The open reading frame (orf) of LmxMPK4 is comprised of 1089 bp coding for a protein of 363 amino acids and a calculated molecular mass of $41.5 \mathrm{kDa}$ [6]. LmxMPK4 is highly conserved in the different Leishmania species (L. panamensis $98.6 \%, L$. donovani $97.5 \%$, L. infantum $97.5 \%$, and $L$. major $98.9 \%$ amino acid identities) and has homologues in other kinetoplastids, like Trypanosoma brucei (67.2\%; E 
A

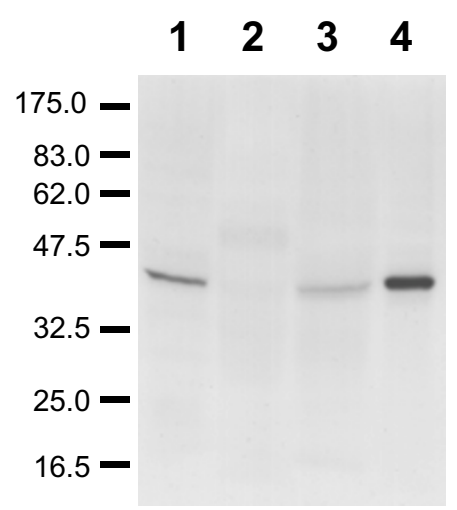

B

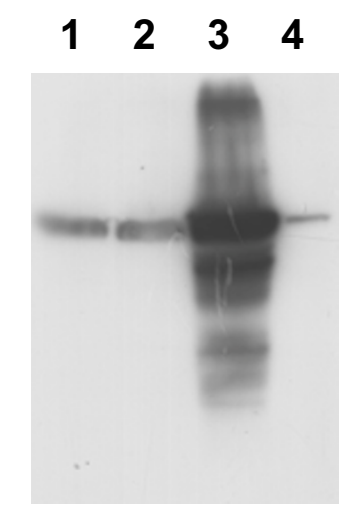

Figure 3

Immunoblot of LmxMPK4 from $L$. mexicana wild type and episomal overexpressor. Lane I, L. mexicana wild type promastigotes ( $4 \times 10^{7}$ cells); lane 2 , amastigotes derived from mouse lesion $\left(4 \times 10^{7}\right.$ cells); lane 3 , wild type in vitro differentiated amastigotes $\left(1 \times 10^{8}\right.$ cells); lane 4, promastigotes of LmxMPK4 episomal overexpressor ( $\mathrm{I} \times 10^{7}$ cells). $A$, blot probed with antiserum against the carboxy-terminal peptide of LmxMPK4; B, the blot was stripped and re-probed with a polyclonal antiserum against myo-inositol-I-phosphate synthase. The molecular masses of standard proteins are indicated in kilodaltons.

value of $\left.5 \mathrm{e}^{-139}\right)$ and T. cruzi (67.4\%; E value of $\left.2 \mathrm{e}^{-143}\right)$, in Chlamydomonas rheinhardtii (43.9\%; E value of $6 \mathrm{e}^{-82}$ ), and in Dictyostelium discoideum (45.0\%; E value of $\left.1 \mathrm{e}^{-85}\right)$. Moreover, it shows weak homology to the human MAP kinases ERK1 (42\%; E value of $1 e^{-70}$ ) and ERK2 (42\%; E value of $\left.6 \mathrm{e}^{-71}\right)$. LmxMPK4 contains the twelve kinase subdomains and amino acid residues known to be highly conserved in MAP kinases (Fig. 1). The kinase domain reaches from tyrosine 17 to phenylalanine 318 encompassing nearly the entire protein leaving only a sixteen residue amino-terminal and a 45 residue carboxy-terminal region. The latter region reveals a potential common docking (CD) domain (DAAEE), a region enriched for negatively charged residues involved in the binding of interacting proteins [7]. Subdomain I contains the phosphate anchor ribbon for ATP binding with the consensus (GXGXXG). A conserved lysine residue (K59), which is involved in orienting ATP for proper phosphate transfer is located in subdomain II. The TXY motif carrying a glutamine in its centre in LmxMPK4, and the P+1 specificity pocket, both hallmarks for MAP kinases are present in subdomain VIII. Finally, a number of other conserved residues are found in the different subdomains. Southern blot analysis of genomic DNA from L. mexicana showed that LmxMPK4 is a single copy gene in the haploid

genome (Fig. 2). PstI cuts within the DNA fragment used as a probe and thus showed two hybridizing bands whereas all other restriction endonucleases only revealed a single band.

\section{Immunoblot analysis}

Using a polyclonal antiserum raised in rabbits against a carboxy-terminal peptide of LmxMPK4, the protein could be detected in a lysate of $4 \times 10^{7}$ wild type promastigotes after lipid extraction, but was hardly detectable in the same number of lesion-derived amastigotes (Fig. 3, lanes 1 and 2). However, we could detect it in in vitro differentiated and cultivated amastigotes which had been under amastigote growth conditions for ten passages. Even here $1 \times 10^{8}$ cells had to be extracted and loaded onto the gel to generate a faint band indicating that the protein is down-regulated in the amastigotes (Fig. 3A, lane 3). On the other hand, we could easily overexpress LmxMPK4 from an episome in promastigotes leading to an at least tenfold overexpression (Fig. 3A, lane 4). To demonstrate differential loading of protein amounts the blot was stripped and re-probed with an antiserum against myoinositol-1-phosphate synthase, a protein known to be equally expressed in promastigotes and amastigotes [8].

\section{Expression of recombinant LmxMPK4 and kinase assay}

To generate an enzymatically inactive KM-mutant LmxMPK4 was mutated changing lysine 59 to methionine. Both, the wild type and the mutant protein, were subcloned into pGEX-KG [9] and expressed as recombinant glutathione S-transferase fusion proteins in Escherichia coli. Both proteins resulted in the same pattern of bands after enrichment on glutathione-sepharose, separation on SDS-polyacrylamide gels and staining with Coomassie brilliant blue. The main contaminating bands were the glutathione S-transferase fusion partner (not shown), a less abundant protein of a higher molecular mass, and a second band just below the GSTLmxMPK4 fusion protein (Fig. 4A, lanes 1 and 2). The latter contaminations were also found while expressing unrelated MAP kinases from L. mexicana in E. coli and therefore are likely of bacterial origin (data not shown). In kinase assays we could detect autophosphorylation of LmxMPK4, but not of the KM-mutant (Fig. 4A, lanes 1 ' and 2') indicating that the phosphorylation of LmxMPK4 is not due to a bacterial kinase which might have been co-purified. However, the kinase activity was relatively weak as films had to be exposed for up to three days to obtain the results shown in figure 4. Phosphorylation of myelin basic protein (MBP) could be obtained with some preparations of recombinant LmxMPK4, but not others (data not shown). We usually perform kinase assays in the presence of 10 $\mathrm{mM} \mathrm{Mg}^{2+}$ and $2 \mathrm{mM} \mathrm{Mn}^{2+}$ at $27^{\circ} \mathrm{C}$. It turned out that manganese on its own in a concentration of $10 \mathrm{mM}$ worked best in kinase assays for LmxMPK4 (Fig. 4B). 


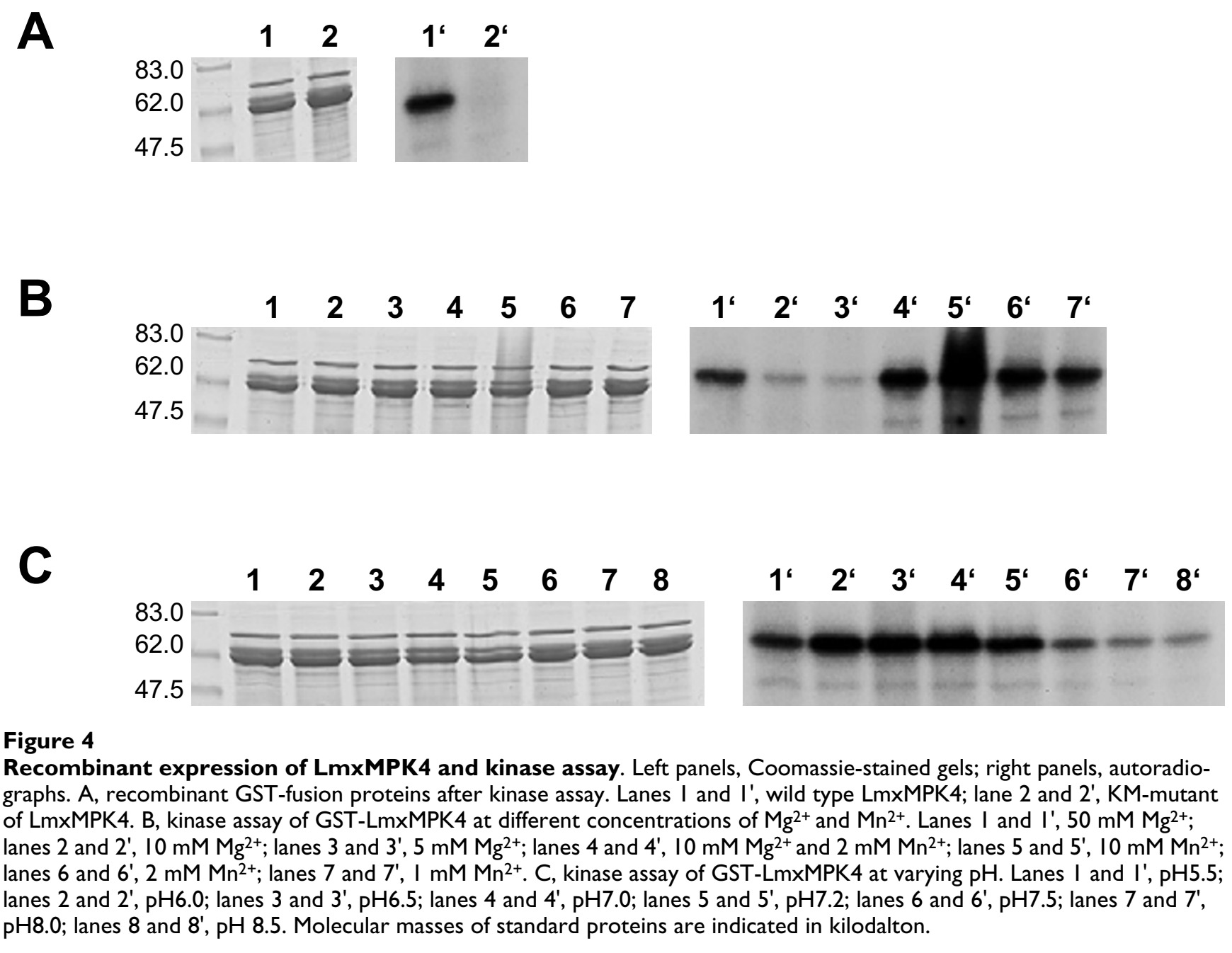

Finally, a pH between 6.0 and 7.0 showed highest autophosphorylation activity in the assay (Fig. 4C).

\section{Deletion analysis of LmxMPK4}

To investigate the function of LmxMPK4 in Leishmania parasites we attempted to obtain homozygous deletion mutants. In order to delete both alleles of the single copy gene we generated constructs comprised of the 5'- and 3'untranslated regions (UTRs) of $\operatorname{LmxMPK4}$ flanking the hygromycin B phosphotransferase gene or the gene of the phleomycin binding protein. Following first rounds of homologous recombination we obtained single-allele deletion mutants for all constructs (data not shown). All our attempts to generate a null mutant for $L m x M P K 4$ resulted in clones carrying the resistance marker genes in the expected genomic context, however, they all retained an extra copy of $L m x M P K 4$ (data not shown). This result seemed to be indicative for a gene which is required for the promastigote life stage of L. mexicana. Therefore, we decided to first add an episomal copy of the LmxMPK4 wild type gene into the promastigotes using the plasmid pXPACLmxMPK4. The presence of the plasmid and the degree of expression of the MAP kinase was checked by Southern and immunoblot analyses (data not shown; Fig. 2, lane 4). Next we repeated our deletion strategy, this time leading to a successful replacement of the genomic alleles of $\operatorname{LmxMPK4}$ in one clone (Fig. 5, lane 5), whereas most of the recombinant parasites retained a genomic allele of LmxMPK4 despite the presence of the gene on the episome (an example is shown in Fig. 5, lane 4). The resulting genomic null mutant parasites showed no obvious phenotype compared to the wild type promastigotes. We used this cell line to infect female Balb/c mice and monitored lesion development for more than 1.5 years. All mice injected showed lesion development (data not shown). Towards the end of the experiment we either sacrificed the mice and isolated the lesion-derived amastigotes for transformation to promastigotes, or took aspirates of the lesion for polymerase chain reaction (PCR) analysis to assess the absence or presence of the plasmid carrying 


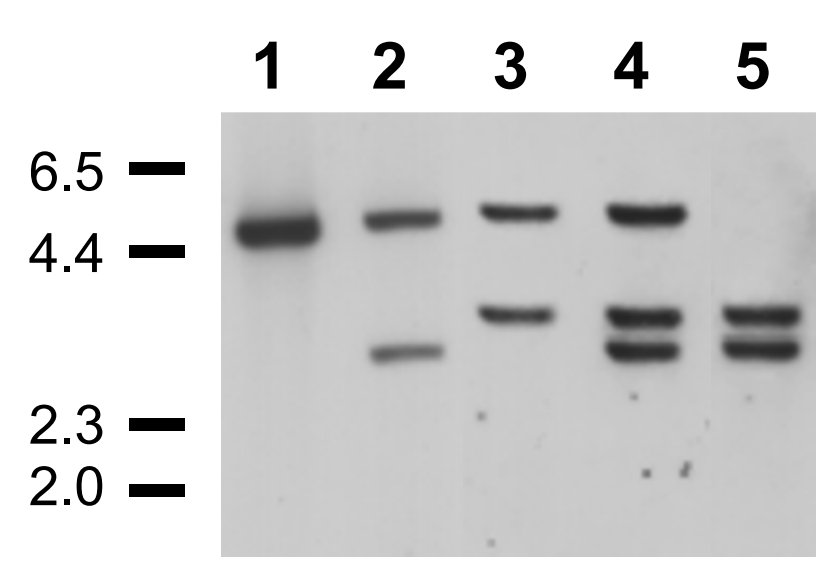

Figure 5

Deletion analysis of LmxMPK4. Southern blot analysis of different clones with a probe corresponding to the 3'-UTR of LmxMPK4. I, L. mexicana wild type; 2, episome pXPACLmxMPK4 + $\Delta L m x M P K 4+/-$ Phleo; 3, episome PXPACLmxMPK4 + $\Delta L m x M P K 4+/-$ Hyg; 4, episome pXPACLmxMPK4 + $\Delta L m x M P K 4-/-/+$ Phleo/Hyg; 5, episome PXPACLmxMPK4 + $\Delta L m x M P K 4-/-$ Phleo/Hyg. Numbers indicate the approximate size of DNA markers in kb.

LmxMPK4. PCR on the total population of freshly differentiated promastigotes proved the presence of $L m x M P K 4$ in the population of cells. Re-isolation of plasmids from freshly differentiated promastigotes and restriction analysis confirmed the identity of the plasmid (data not shown). To evaluate the presence of plasmids in amastigotes we isolated parasites by taking lesion aspirates. As a control wild type amastigotes were isolated and treated like the mutant amastigotes. The amastigotes were liberated from their host cells by shear forces also disrupting aggregates of amastigotes leading to a suspension of single cells. The DNA of the cells was stained using SYTO16 (Invitrogen, Heidelberg, Germany) followed by sorting of the parasites into 96-well plates. A duplex PCR was performed using a pair of oligonucleotide primers specific for the single-copy gene LmxMPK9, which generates a 254 bp DNA fragment, and a second pair of oligonucleotides specific to detect the presence of $L m x M P K 4$ by a 420 bp DNA fragment. In a series of PCRs using different numbers of sorted wild type amastigotes it turned out that at least ten amastigotes per well had to be used to give reliable results in the subsequent PCR. Only one reaction out of 44 failed to show the control fragment for $L m x M P K 9$ in the mutant. This rare event of no amplification was not found for $L m x M P K 9$ in the wild type but was observed for $L m x M P K 4$ in both wild type and mutant amastigotes indicating that it is indeed a PCR failure and the plasmid is definitely present in all cells. Looking at freshly differentiated promastigotes we obtained a significantly different picture. As promastigotes could be purified much easier we suc- ceeded to perform the duplex PCR on the single cell level with high efficiency. Again a failure of the PCR could be observed, as in 5\% of all wells (4/77) the control fragment was not amplified. The percentage of wells lacking the LmxMPK4 fragment was 27\% (21/77). This higher percentage is indicative for the presence of single cells lacking the plasmid and thus the gene. As this gives no clue about the ability of those promastigotes having lost the plasmid to proliferate, we cloned the cells by serial dilution and analysed the populations arising from single cells using the duplex PCR. All 66 clones obtained still retained the plasmid. Therefore, LmxMPK4 is essential for promastigotes and amastigotes.

\section{Discussion}

LmxMPK4 has been found to be a MAP kinase homologue from L. mexicana by amino acid sequence comparison and an active protein kinase by proving the activity of the recombinant GST-fusion protein in in vitro kinase assays. The protein was hardly detectable in amastigotes by immunoblot analysis, however, the fact that amastigotes lacking the genomic copies of $L m x M P K 4$ retained a plasmid carrying the gene without any antibiotic selection imposed on the cells while in the mouse for more than 1.5 years strongly suggests that the protein is expressed and essential for this life stage. Moreover, all attempts to generate homozygous deletion mutants in the promastigotes failed and constantly ended in parasites showing accurate replacement of both alleles of $\operatorname{LmxMPK4}$, but an additional copy of the gene which might reflect a chromosomal duplication. Thus, LmxMPK4 is required in the insect stage promastigotes as well. This is contrary to TbMAPK2, the T. brucei homologue of LmxMPK4, which could be deleted in the mammalian stage bloodstream form trypanosomes without a detectable phenotype [10]. However, differentiation of the null mutant to procyclic trypanosomes by temperature shift from $37^{\circ} \mathrm{C}$ to $27^{\circ} \mathrm{C}$ and addition of cis-aconitate revealed delayed differentiation kinetics and an arrest of the parasites in all phases of the cell cycle. It is likely, that in T. brucei procyclic forms cell cycle progression depends on a constitutive stimulus through TbMAPK2 signalling. In Leishmania the importance of LmxMPK4 is even extended to the mammalian stage of the parasite making this kinase a potential drug target to treat Leishmaniasis.

\section{Conclusion}

LmxMPK4 is a mitogen-activated protein kinase homologue from L. mexicana, that is highly conserved throughout different Leishmania species and is essential for the two main proliferating life stages, the promastigotes and amastigotes, of the parasite. Moreover, it reveals significant amino acid divergence compared to MAP kinases from mammals showing highest homology to protein kinases from kinetoplastids, low homology to human 
kinases and kinases from Chlamydomonas and Dictyostelium. Therefore, it represents a suitable and promising drug target to prevent the proliferation of the parasites and cure the disease.

\section{Methods \\ Parasites}

Promastigotes of L. mexicana MNYC/BZ/62/M379 were grown as described [11]. Female Balb/c mice were infected into the left hind foot pad at the age of 6-8 weeks with 1 $\times 10^{7}$ promastigote parasites in $30 \mu \mathrm{l}$ PBS $(137 \mathrm{mM} \mathrm{NaCl}$, $2.7 \mathrm{mM} \mathrm{KCl}, 8 \mathrm{mM} \mathrm{Na}_{2} \mathrm{HPO}_{4}, 1.4 \mathrm{mM} \mathrm{KH}_{2} \mathrm{PO}_{4}$ ). Infection experiments have been approved by the Veterinary Authorities of the Freie and Hansestadt Hamburg and were performed in accordance with section 8a paragraph 1 together with section 10a of the German Animal Protection Law as amended on May 25, 1998, with latest amendment from August 6, 2002 (GZ.: G 21132/59100.33). Using a caliper gage, the course of infection was followed by measuring lesion size relative to the uninfected right hind footpad at 4-5 weeks intervals. Amastigotes were isolated from lesions of Balb/c mice as described [5]. For small scale amastigote preparation the lesion was aspirated 2-3 times with a $23 \mathrm{G} \times 11 / 4$ " needle and a syringe. The isolated tissue was transferred into $0.5 \mathrm{ml}$ of cold SYTO16-buffer (21 mM Hepes pH 7.5, $137 \mathrm{mM}$ $\mathrm{NaCl}, 5 \mathrm{mM} \mathrm{KCl}, 6 \mathrm{mM}$ Glucose) and kept on ice. The tissue was passed five times through a $30 \mathrm{G} \times 1 / 2$ " needle to generate single cells. $850 \mu \mathrm{l}$ of cold SYTO16-buffer, $150 \mu \mathrm{l}$ DMSO and $0.5 \mu \mathrm{l}$ of $33 \mu \mathrm{M}$ SYTO16 (Invitrogen) in DMSO were added, the cells mixed and incubated for 30 minutes at room temperature in the dark. Afterwards the cells were washed twice with SYTO16-buffer and were finally resuspended in $1 \mathrm{ml}$ SYTO16-buffer and stored at $4^{\circ} \mathrm{C}$ in the dark before sorting. For promastigote staining $1.1 \times 10^{8}$ cells were taken from a log-phase culture, washed twice with $1 \mathrm{ml}$ SYTO16-buffer, and processed as described above.

\section{Gene cloning, sequencing and analysis}

Positive phage clones for LmxMPK4 were selected using digoxigenin (DIG)-labelled probes [6], amplified and DNA inserts cloned into pBSKII(+) using EcoRI (Stratagene, La Jolla, CA) yielding pBE25LmxMPK4. A 4514 bp ClaI DNA fragment was liberated from pBE25LmxMPK4 and ligated into pBSKII(+) yielding pB2CLmxMPK4. Both DNA strands were sequenced. Plasmid isolation, DNA sequencing and analysis, DNA isolation and blotting, and hybridizations were performed basically as described before [12]. A probe encompassing nt 204 - nt 930 of the orf of LmxMPK4 was DIG-labelled using the oligonucleotides 5'-TGCGGGAGATGGAGATAA-3' and 5'-CTTCGCAGTTAATCGTCTT-3' and the PCR DIG probe synthesis kit as described by the manufacturer (Roche, Mannheim, Germany). For labelling of the 2350 bp SphI/NruI DNA fragment corresponding to the 3 ' untranslated region of $L m x M P K 4$ the fragment was isolated from pB2CLmxMPK4 and labelled using the DIG DNA labelling kit (Roche). LmxMPK4 sequence data have been submitted to the DDBJ/EMBL/GenBank databases under the accession number $\underline{\mathrm{AJ} 293282}$.

\section{LmxMPK4 deletion constructs}

The Expand ${ }^{\mathrm{TM}}$ High Fidelity PCR or the Expand Long Template PCR System (Roche) was used for all polymerase chain reaction (PCR) applications. To generate the construct for a genomic allele deletion mutant for $L m x M P K 4$ the flanking regions were amplified by PCR from pB2CLmxMPK4 cut with BglII using 5'-GCTGAGCCATGGTTCTCTATGCCTCC-3' and 5'-TGAAAGCCTAGGAATCTGGTGCTCTC-3' $\left(3 \mathrm{~min} 94^{\circ} \mathrm{C}, 10 \times\left[20 \sec 94^{\circ} \mathrm{C}\right.\right.$, $\left.30 \mathrm{sec} 45^{\circ} \mathrm{C}, 7 \min 68^{\circ} \mathrm{C}\right], 20 \times\left[20 \mathrm{sec} 94^{\circ} \mathrm{C}, 30 \mathrm{sec}\right.$ $45^{\circ} \mathrm{C}, 7 \mathrm{~min}+20 \mathrm{sec} /$ cycle $68^{\circ} \mathrm{C}$ ], $7 \mathrm{~min} 68^{\circ} \mathrm{C}, 4^{\circ} \mathrm{C}$ ). The gel-purified PCR-fragment was trimmed at the ends using $\mathrm{NcoI}$ and AvrII, and ligated to a BspHI/NheI fragment containing either the phleomycin binding protein gene (BLE) or the hygromycin B phosphotransferase gene (HYG) as described before [12], yielding pB11C $\Delta$ LmxMPK4phleo and pB21C $\Delta$ LmxMPK4hyg. Both constructs were cut with ClaI and NruI, the DNA fragments for homologous recombination were gel-purified and used for electroporation in two consecutive rounds and recombinants selected in medium containing $5 \mu \mathrm{g} \mathrm{ml}{ }^{-1}$ bleocin and $20 \mu \mathrm{g} \mathrm{ml}^{-1}$ hygromycin B [4].

\section{Episomal expression constructs for LmxMPK4}

For episomal expression of $L m x M P K 4$ the orf was amplified from pB2CLmxMPK4 using 5'-CCCGATATCATGACTCAGCTCGTCCC-3' and 5'CCCGATATCTCGAGCCTATTCGTTCAATTGTG-3' (5 min $94^{\circ} \mathrm{C}, 20 \times\left[30 \sec 94^{\circ} \mathrm{C}, 30 \sec 45^{\circ} \mathrm{C}, 1 \min 72^{\circ} \mathrm{C}\right], 7$ min $72^{\circ} \mathrm{C}, 4^{\circ} \mathrm{C}$ ) introducing EcoRV, BspHI, and XhoI restriction sites, trimmed with EcoRV and ligated into pBSKII(+) to yield pB6ELmxMPK4. Both DNA strands were sequenced. The 1104 bp EcoRV fragment carrying LmxMPK4 was liberated and cloned into pX63polPAC linearised at its single EcoRV site [4]. Cells were transfected with $20 \mu \mathrm{g}$ of the plasmid constructs designated pXPACLmxMPK4 as described above, and transformants were selected in SDM-79 using $20 \mu \mathrm{M}$ puromycin.

\section{Expression constructs, mutagenesis and antibody production}

The $1095 \mathrm{bp} B s p \mathrm{HI} / \mathrm{XhoI}$ DNA fragment was isolated from pB6ELmxMPK4, ligated into pGEX-KG [9] cut with NcoI and $X h o I$, and the resulting construct was transformed into E. coli XL1-blue. Expression and purification of the glutathione-S-transferase fusion protein was performed as described before [4]. To generate an enzymatically inactive version of LmxMPK4 lysine 59 was replaced by 
methionine. The oligonucleotides 5'-GTTAGACTCGCGACAACCGCATGATAGCCACTCGCT-

CACC-3' introducing NruI sites and the desired mutation were used in a PCR on pB6ELmxMPK4 $\left(94^{\circ} \mathrm{C}, 5 \mathrm{~min} ; 10\right.$ $\times\left[94^{\circ} \mathrm{C}, 30 \mathrm{~s} ; 45^{\circ} \mathrm{C}, 30 \mathrm{~s} ; 68^{\circ} \mathrm{C}, 3 \mathrm{~min}\right] ; 10 \times\left[94^{\circ} \mathrm{C}, 30\right.$ $\mathrm{s} ; 55^{\circ} \mathrm{C}, 30 \mathrm{~s} ; 68^{\circ} \mathrm{C}, 3 \mathrm{~min}+20 \mathrm{~s} /$ cycle]; $68^{\circ} \mathrm{C}, 7 \mathrm{~min}$; $4^{\circ} \mathrm{C}$ ). The amplified fragment was gel-purified, trimmed at the ends using NruI, circularised by self-ligation, and transformed into E. coli. Successful mutagenesis was confirmed by sequencing. Finally, the mutated gene was cloned into pGEX-KG as described above. A rabbit antiserum was produced against the peptide CEDLWRVVEAHSQLNE corresponding to the 15 carboxy-terminal amino acids of LmxMPK4 (Eurogentec, Seraing, Belgium).

\section{Immunoblotting}

Cells were harvested by centrifugation and washed once using PBS. The pellet was resuspended in chloroform/ methanol/ $\mathrm{ddH}_{2} \mathrm{O}(1: 2: 0.8)$ in a concentration of $2 \times 10^{8}$ cells $/ \mathrm{ml}$ and left at room temperature for $30 \mathrm{~min}$. The extracted cells were pelleted for $10 \mathrm{~min}$ at $15000 \times \mathrm{g}$ at room temperature and the pellet dried under vacuum (speed-vac). Then the pellet was resuspended in $100 \mu \mathrm{l} 50$ $\mathrm{mM}$ Tris/HCl $\mathrm{pH}$ 8.0, $20 \mu \mathrm{M}$ leupeptin, $1 \mathrm{mM}$ phenylmethylsulfonyl fluoride, $5 \mathrm{mM}$ 1,10-phenanthroline, 10 $\mathrm{mM} \mathrm{MgCl} 2,100 \mathrm{U} / \mathrm{ml}$ benzonase (Merck, Darmstadt, Germany) and incubated at $37^{\circ} \mathrm{C}$ for $30 \mathrm{~min}$. Dithiothreitol (DTT) was added to a final concentration of $50 \mathrm{mM}$ and the probe adjusted to $1 \times$ SDS sample buffer $(0.4 \%$ SDS, $4 \%$ glycerol, $0.0002 \%$ bromphenol blue, $50 \mathrm{mM}$ DTT, $12.5 \mathrm{mM}$ Tris-HCl $\mathrm{pH}$ 6.8). Probes were boiled for 10 min, 20-40 $\mu \mathrm{l}$ subjected to SDS-polyacrylamide gel electrophoresis (PAGE) and blotted to poly(vinylidenedifluoride) (PVDF) membranes. Immunodetection was carried out as described before [5] using the polyclonal rabbit antiserum and goat-anti-rabbit secondary antibodies coupled to peroxidase (Dianova, Hamburg, Germany) followed by chemiluminescence using the ECL system (Amersham Pharmacia Biotech, Freiburg, Germany).

\section{Kinase assay}

$1 \mu \mathrm{g}$ of the purified kinase was used in $50 \mu \mathrm{l}$ kinase assay solution (50 mM Tris pH 7.5, $0-50 \mathrm{mM} \mathrm{MgCl}_{2}, 0-10 \mathrm{mM}$ $\mathrm{MnCl}_{2}, 0.1 \mathrm{M} \mathrm{NaCl}, 5 \mu \mathrm{Ci}\left(\gamma_{-32}{ }^{32}\right)$-ATP, $1 \mathrm{mM}$ ATP, $5 \mu \mathrm{g}$ myelin basic protein) and incubated at $27^{\circ} \mathrm{C}$. The different $\mathrm{pH}$ conditions tested were reached using $50 \mathrm{mM}$ morpholinoethane sulfonic acid for pH6.5 and below, 3-(Nmorpholino)propanesulfonic acid for $\mathrm{pH} 7$ and 7.2, and $50 \mathrm{mM}$ Tris/HCl for $\mathrm{pH} 7.5$ and higher. $12.5 \mu \mathrm{l} 5 \times$ SDS sample buffer were added and the solution heated for 10 min at $95^{\circ} \mathrm{C}$. $25 \mu \mathrm{l}$ of the solution were separated on a $12 \%$ SDS-PAGE, stained with Coomassie brilliant blue, destained, dried and exposed to X-ray films at $-70^{\circ} \mathrm{C}$.

\section{Cell sorting and PCR analysis}

Single promastigotes stained with SYTO16 were gated with a forward scatter FSC-A $<80$ and a side scatter SSC-A $<80$ and sorted into skirted 96-well PCR plates (Sarstedt, Nuembrecht, Germany) using a FACSAria (Becton \& Dickinson, Franklin Lakes, NJ). For lesion-derived amastigotes the smallest SYTO16-positive particles (FSC-A $<50$, SSC$A<50$ ) were sorted with ten cells per well. All PCR reactions were performed in an Eppendorf Mastercycler ep (Eppendorf, Hamburg, Germany). $25 \mu \mathrm{l}$ of a mastermix were added to each well that contained pro- or amastigotes. $24 \mu \mathrm{l}$ of mastermix and $1 \mu \mathrm{l}$ of genomic DNA (c = $0.24 \mu \mathrm{g} / \mu \mathrm{l}$ ) of $L$. mexicana served as a positive control, as a negative control $25 \mu \mathrm{l}$ of mastermix were used. After addition of the mastermix the plates were sealed with adhesive foil (Sarstedt). $0.5 \mu \mathrm{l}$ AccuPrime Taq DNA Polymerase (Invitrogen) were used in a volume of $25 \mu \mathrm{l}$ with $5 \mathrm{pmol}$ of the respective oligonucleotide primers (10 $\min 94^{\circ} \mathrm{C}, 45 \times\left[10 \sec 94^{\circ} \mathrm{C}, 15 \sec 60^{\circ} \mathrm{C}, 45 \sec 68^{\circ} \mathrm{C}\right.$, $7 \mathrm{~min} 68^{\circ} \mathrm{C}, 4^{\circ} \mathrm{C}$ ). For the amplification of the $254 \mathrm{bp}$ control DNA fragment derived from LmxMPK9 [6] we used 5'-GTCAGCGTGCCAATGAAAT-3' and 5'CAAGCTCCGGTGCGCGGTA-3'. A 420 bp DNA fragment corresponding to $L m x M P K 4$ is amplified by 5 '-TATGATTCAAGCATTCCGCG-3' and 5'-CGGCCGATGCGGCTGAGAG-3'. The samples were analysed on $1.5 \%$ agarose gels.

\section{Competing interests}

The author(s) declare that they have no competing interests.

\section{Authors' contributions}

Q.W. cloned and sequenced LmxMPK4, constructed the deletion and overexpression constructs, and performed the deletion analysis and immunoblotting experiments.

I.M.M. expressed and purified the recombinant proteins and performed the enzymatic characterisation.

M.K. isolated amastigotes, prepared cells for FACS sorting and performed PCR analysis on sorted cells.

C.S.-J. performed the cell sorting on a Becton Dickinson FACSAria.

M.W. conceived the study, supervised the execution, did PCR analysis on cloned cell lines, and prepared the final draft of the manuscript.

All authors read and approved the final manuscript.

\section{Acknowledgements}

We thank Anne Scholz for critically reading the manuscript, the Gilberger group at the Bernhard Nocht Institute for Tropical Medicine for advice and letting us use their Eppendorf Mastercycler, Iris Goercke for expert tech- 
nical assistance, and Thomas Ilg (Tuebingen) for providing the myo-inositolI-phosphate synthase antiserum.

\section{References}

I. Waskiewicz AJ, Cooper JA: Mitogen and stress response pathways: MAP kinase cascades and phosphatase regulation in mammals and yeast. Curr Opin Cell Biol 1995, 7:798-805.

2. Parsons M, Worthey EA, Ward PN, Mottram JC: Comparative analysis of the kinomes of three pathogenic trypanosomatids: Leishmania major, Trypanosoma brucei and Trypanosoma cruzi. BMC Genomics 2005, 6: 127.

3. Wiese M, Kuhn D, Grunfelder CG: Protein kinase involved in flagellar-length control. Eukaryot Cell 2003, 2:769-777.

4. Bengs F, Scholz A, Kuhn D, Wiese M: LmxMPK9, a mitogen-activated protein kinase homologue affects flagellar length in Leishmania mexicana. Mol Microbiol 2005, 55:1606-1615.

5. Wiese M: A mitogen-activated protein (MAP) kinase homologue of Leishmania mexicana is essential for parasite survival in the infected host. EMBO | 1998, 17:2619-2628.

6. Wiese M, Wang Q, Gorcke I: Identification of mitogen-activated protein kinase homologues from Leishmania mexicana. Int J Parasitol 2003, 33:1577-1587.

7. Tanoue T, Nishida E: Molecular recognitions in the MAP kinase cascades. Cell Signal 2003, 1 5:455-462.

8. IIg T: Generation of myo-inositol-auxotrophic Leishmania mexicana mutants by targeted replacement of the myoinositol-I-phosphate synthase gene. Mol Biochem Parasitol 2002, 120:15I-156.

9. Guan KL, Dixon JE: Eukaryotic proteins expressed in Escherichia coli: an improved thrombin cleavage and purification procedure of fusion proteins with glutathione S-transferase. Anal Biochem 1991, 192:262-267.

10. Muller IB, Domenicali-Pfister D, Roditi I, Vassella E: Stage-specific requirement of a mitogen-activated protein kinase by Trypanosoma brucei. Mol Biol Cell 2002, 13:3787-3799.

II. Menz B, Winter G, Ilg T, Lottspeich F, Overath P: Purification and characterization of a membrane-bound acid phosphatase of Leishmania mexicana. Mol Biochem Parasitol 1991, 47:101-108.

12. Benzel I, Weise F, Wiese M: Deletion of the gene for the membrane-bound acid phosphatase of Leishmania mexicana. Mol Biochem Parasitol 2000, I I I:77-86.

13. Gaskins C, Maeda M, Firtel RA: Identification and functional analysis of a developmentally regulated extracellular signalregulated kinase gene in Dictyostelium discoideum. Mol Cell Biol 1994, 14:6996-70I2.

14. Gonzalez FA, Raden DL, Rigby MR, Davis RJ: Heterogeneous expression of four MAP kinase isoforms in human tissues. FEBS Lett 1992, 304: 170-178.

\section{Publish with Bio Med Central and every scientist can read your work free of charge}

"BioMed Central will be the most significant development for disseminating the results of biomedical research in our lifetime. "

Sir Paul Nurse, Cancer Research UK

Your research papers will be:

- available free of charge to the entire biomedical community

- peer reviewed and published immediately upon acceptance

- cited in PubMed and archived on PubMed Central

- yours - you keep the copyright

Submit your manuscript here:

http://www.biomedcentral.com/info/publishing_adv.asp
BiolMedcentral 